\title{
Shale Gas Productivity Predicting Model and Analysis of Influence Factors
}

\author{
Yin Daiyin, Wang Dongqi", Zhang Chengli and Duan Yingjiao \\ Northeast Petroleum University, Key Laboratory of Enhanced Oil and Gas Recovery of Ministry of Education, Daqing, \\ P.R. China
}

\begin{abstract}
In order to find the dynamic characteristics of shale gas reservoirs and improve shale gas well production, it is very important to research on shale gas seepage mechanism and production evaluation. Based on the shale gas seepage mechanism, adsorption and desorption characteristics, the diffusion mechanism and mass conservation theory in shale gas development, the dual pore medium shale gas reservoir mathematical model is set up. The mathematical model is built by the finite difference method based on start-up pressure gradient, slippage effect and the isothermal adsorption principle, and then programmed to solve it. Finally, this paper analyzed the impact of Langmuir volume, Langmuir pressure, start-up pressure gradient and slippage coefficient and other factors on shale gas wells production.
\end{abstract}

Keywords: Influence factors, Seepage mechanism, Shale gas, Slippage effect, Start-up pressure gradient.

\section{INTRODUCTION}

The exploration and development of the shale gas, a kind of unconventional oil and gas resources [1-3], is getting more and more attention in the oil industry. Global shale gas resource is about $456.24 \times 10^{12} \mathrm{~m}^{3}$. Shale gas resources in our China is very abundant, which is estimated to be about $26 \mathrm{x}$ $10^{12} \mathrm{~m}^{3}$ by Zhang Jinchuan. Shale gas is an unconventional gas generated by mudstone and shale under various geological conditions, and is mainly reserved in free and adsorption states, which has led to the rise of high quality clean energy in recent years. The occurrence forms include adsorption, free and dissolved states, and the shale gas in adsorption state accounts for $20 \% \sim 80 \%$ of the total shale gas [4], therefore the research on seepage mechanism and migration characteristics is very significant for effective exploitation of shale gas. Shale gas production research can predict shale gas well production, determine production dynamic characteristics, and optimize well pattern arrangement and completion program, which lays the foundation of gas well proration and further study on gas reservoir engineering. Considering start-up pressure gradient, slippage effect, adsorption and desorption characteristics, seepage mechanism and the law of conservation of mass, finite difference method was used to build numerical model and the corresponding program was executed to solve it, and then various factors impacting shale gas production were analyzed [5].

\section{SEEPAGE MECHANISM}

Shale gas reservoirs are typical self-generated and selfstored reservoirs, and the main occurrence forms of shale gas are adsorption and free states [6,7]. Shale gas in adsorption states mainly reserves in the surface of clay and organic matter particles, and shale gas in free states reserves in shale matrix pores and fractures. However, the dissolved gas con-

\footnotetext{
*Address correspondence to this author at the Northeast Petroleum University, Key Laboratory of Enhanced Oil and Gas Recovery of Ministry of Education, Daqing, P.R. China; Tel/Fax: +86-138-4594-8796;

E-mails: yindaiyin@163.com,dbwdq@126.com.
}

tent is less, dissolved in kerogen and asphaltene. Shale reservoir, a porous medium, mainly consists of four parts, respectively no-organic substrate, organic kerogen, natural fracture and hydraulic fracturing cracks.

Shale gas flow mechanism mainly includes the following three processes [8-10]: (1) when the formation pressure drops to desorption pressure in the development, gas desorbs step by step from shale substrate surface and becomes free gas; (2) as the shale gas desorbs, internal and surface concentration difference is generated between interior and surface of matrix system, and desorption gas enters into natural or hydraulic fracturing cracks by diffusion; (3) gas seeps through the natural and artificial fracturing cracks, eventually, into the wellbore.

\subsection{Mathematical Model Establishment}

To set up the shale gas seepage mathematical model, the following basic assumptions are needed: $\ominus$ the shale gas reservoir is a dual porosity reservoir with uniform physical properties. $\ominus$ rock and gas can be compressed; $\circledast$ the temperature at each point is constant in the shale gas reservoir, i.e. the seepage is an isothermal process; (4) the flow is singlephase gas flow with negligible gravity and capillary force.

(1) The equations of motion considering start-up pressure gradient and slippage effect

In low permeability and low porosity shale gas reservoirs, the gas seepage is not in conformity with the Darcy law. Besides viscous resistance, there exists absorption resistance. When pressure gradient is higher than the start-up pressure gradient, gas begins to flow; otherwise the flow does not occur; when pressure gradient is lower, it will deviate from being linear. The following is a simplified model considering start-up pressure gradient $[11,12]$ :

$$
\begin{cases}v=0 & \left|\frac{\partial P}{\partial r}\right| \leq \eta \\ v=-\frac{k}{\mu}\left(\frac{\partial P}{\partial r}-\eta\right) & \left|\frac{\partial P}{\partial r}\right|>\eta\end{cases}
$$


Seepage velocity influenced by slipping effect:

$v=-\left(\frac{86.4 K_{f}}{\mu} \Delta p_{f}\right)\left(1+\frac{b}{\bar{p}}\right)$ ty:

Combining type (1) with type (2) can get seepage veloci-

$v=-\left(\frac{86.4 K_{f}}{\mu}\left(\Delta p_{f}-\eta\right)\right)\left(1+\frac{b}{p}\right)$

(2)Shale gas absorption equation [13]

Isothermal adsorption equation:

$V_{E}=\frac{V_{L} P_{g}}{P_{L}+P_{g}}$

The diffusion equation:

$\frac{\partial V_{m}}{\partial t}=-D_{m} F_{s}\left(V_{m}-V_{E}\right)=-\frac{1}{\tau}\left(V_{m}-V_{E}\right)$

Diffusion amount equation:

$q_{m}=-F_{G} \frac{\partial V_{m}}{\partial t}$

(3) The equation of state of shale gas $[14,15]$

$\rho_{g}=\frac{p M}{Z R T}$

Gas compressibility:

$C_{g}=-\left.\frac{1}{V_{g}} \frac{d V_{g}}{d P}\right|_{T}=\frac{1}{P}-\left.\frac{1}{z} \frac{d z}{d P}\right|_{T}$

Pore compressibility:

$C_{f}=-\frac{1}{\phi} \frac{d \phi}{d P}$

Comprehensive compressibility:

$C_{t}=C_{f}+C_{g}$

(4) The basic seepage equations

The seepage equation of the matrix system:

$-\rho_{g} F_{G} \frac{\partial V_{m}}{\partial t}-\rho_{g} \frac{a k_{m}}{\mu}\left(P_{m}-P_{f}\right)=\frac{\partial\left(\rho_{g} \phi_{m}\right)}{\partial t}$

The seepage equation of the fracture system:

$\frac{1}{r} \frac{\partial}{\partial r}\left(r \rho_{g} v_{f}\right)+\rho_{g} \frac{a k_{m}}{\mu}\left(P_{m}-P_{f}\right)=\frac{\partial\left(\rho_{g} \phi_{f}\right)}{\partial t}$

According to the law of conservation of mass, by integrating all kinds of equations, shale gas seepage equation is obtained: $\frac{1}{r} \frac{\partial}{\partial r}\left(r \rho_{g} \frac{k_{f}}{\mu}\left(\frac{\partial P}{\partial r}-\eta\right)\left(1+\frac{b}{P}\right)\right)=\frac{\partial\left(\rho_{g} \phi_{m}\right)}{\partial t}+$

$\rho_{g} F_{G} \frac{\partial V_{m}}{\partial t}+\frac{\partial\left(\rho_{g} \phi_{f}\right)}{\partial t}$

Initial condition:

$\left.p\right|_{t=0}=p_{i}$

Inner boundary condition:

$p_{w f}=\left(p-S r \frac{\partial p_{f}}{\partial r}\right)_{r=r w}$

Outer boundary condition:

$\left(r \frac{\partial p}{\partial r}\right)_{r=r w}=0$

(5) Finite difference form of seepage equation

Non-equidistantly subdivide the gas reservoir seepage areas, and use block center grid system:

if $\Delta x=\frac{1}{N} \ln \frac{r_{e}}{r_{w}}$, the outer radius of the $i$ piece

$r_{i+\frac{1}{2}}=r_{w} e^{i \Delta x}$, the inner radius $r_{i-\frac{1}{2}}=r_{w} e^{(i-1) \Delta x}$, and the outer boundary $r_{e}=r_{N+\frac{1}{2}}$.

The equation (11) and the equation (12) for each grid are transformed into finite difference forms (17) and (18) respectively. Tri-diagonal equations about fracture system pressure can be obtained through substituting equation (17) into equation (18). Then based on formulas (14), (15) and (16), the well production can be calculated out after the formation pressure is obtained.

Finite difference form of the seepage equation for matrix system:

$$
A_{i}^{n} p_{m i}^{n+1}+B_{i}^{n} p_{f i}^{n+1}=C_{i}^{n} p_{m i}^{n}
$$

Finite difference form of the seepage equation for fracture system:

$d_{i}^{n} p_{f_{i-1}}^{n+1}+e_{i}^{n} p_{f_{i}}^{n+1}+f_{i}^{n} p_{f_{i+1}}^{n+1}=g_{i}^{n} p_{f_{i}}^{n}+h_{i}^{n} p_{m_{i}}^{n}+l_{i}^{n}$

Where,

$A_{i}^{n}=\left(\frac{\phi_{m} c_{t n}}{\Delta t}+F_{G} \frac{P_{L} V_{L}}{2 \Delta t\left(P_{L}+P_{g}\right)^{2}}\left(1-e^{-\frac{\Delta t}{r}}\right)+\frac{3.6 a k_{m}}{\mu}\right)_{i}^{n} B_{i}^{n}=\left(\frac{3.6 a k_{m}}{\mu}\right)_{i}^{n}$

$C_{i}^{n}=\left(\frac{\phi_{m} c_{m}}{\Delta t}+F_{G} \frac{P_{L} V_{L}}{2 \Delta t\left(P_{L}+P_{g}\right)^{2}}\left(1-e^{-\frac{\Delta-}{r}}\right)\right)_{i}^{n}$ 
$d_{i}^{n}=7.2 \delta_{i-\frac{1}{2}}^{n}, \quad f_{i}^{n}=7.2 \delta_{i+\frac{1}{2}}^{n}$

$e_{i}^{n}=-\left(7.2 \delta_{i-\frac{1}{2}}^{n}+7.2 \delta_{i+\frac{1}{2}}^{n}+3.6 \beta_{i}^{n} \frac{a k_{m} C_{i}^{n}}{\mu\left(B_{i}^{n}+C_{i}^{n}\right)}+\frac{\beta_{i}^{n} \phi C_{t i}^{n}}{\Delta t}\right)$

$g_{i}^{n}=-3.6 \beta_{i}^{n} \frac{a k_{m} C_{i}^{n}}{\mu\left(B_{i}^{n}+C_{i}^{n}\right)}, \quad h_{i}^{n}=-3.6 \beta_{i}^{n} \frac{a k_{m} C_{i}^{n}}{\mu\left(B_{i}^{n}+C_{i}^{n}\right)}$,

$l_{i}^{n}=\frac{7.2 \pi k_{f} h \eta}{\mu}\left(1+\frac{b}{\bar{p}}\right)\left(\rho_{i+\frac{1}{2}}-\rho_{i-\frac{1}{2}}\right)$,

$\delta=\frac{\pi k_{f} h \rho}{\mu \Delta x}\left(1+\frac{b}{p}\right), \quad \beta_{i}^{n}=\pi\left(\begin{array}{c}r_{i+\frac{1}{2}}^{2}-r^{2} \\ i-\frac{1}{2}\end{array}\right) h \rho_{i}^{n}$

(6) Production calculation at a certain time

Based on formula (3), we get:

$\frac{q}{172.8 \pi r h}=-\left(\frac{K_{f}}{\mu}\left(\Delta p_{f}-\eta\right)\right)\left(1+\frac{b}{p}\right)$

The integration type of equation (19) is:

$\int_{r_{w}}^{r_{e}}\left(\frac{q}{172.8 \pi r h}+\frac{K_{f} \eta}{\mu}\left(1+\frac{b}{p}\right)\right) d r=\int_{p_{w}^{n}}^{p_{p}^{n}} \frac{K_{f}}{\mu}\left(1+\frac{b}{p}\right) d p$

For stable seepage, the values of $\bar{\mu}$ and $\bar{Z}$ corresponding to the average pressure are taken as the values of viscosity $\mu$ and compressibility factor $Z$. After integration of equation (20), we get:

$\frac{q}{172.8 \pi h} \ln \left(\frac{r_{e}}{r_{w}}\right)=\frac{k_{f}}{\bar{\mu}}\left(1+\frac{b}{{ }_{p}}\right)\left\{\left(p_{e}^{n}-p_{w}^{n}\right)-\eta\left(r_{w}-r_{e}\right)\right\}$

Based on state equation (7), we get:

$q=q_{s c} \frac{p_{s c}}{Z_{s c} T_{s c}} \frac{Z T}{p}$
Combining (1) with (2) can get gas volume flow formula corresponding to standard state:

$q_{s c}=\frac{p^{-n}}{\bar{Z} T} \frac{Z_{s c} T_{s c}}{p_{s c}} \frac{172.8 \pi h k_{f}}{\bar{\mu}} \frac{\left(1+\frac{b}{-\bar{p}}\right)\left\{\left(p_{e}^{n}-p_{w}^{n}\right)-\eta\left(r_{w}-r_{e}\right)\right\}}{\ln \left(\frac{r_{e}}{r_{w}}\right)}$

The dimensionless forms of production and time are:

$$
q_{s c D}^{n}=\frac{q_{s c}^{n}}{q_{s c i}}, \quad t_{D}^{n}=\frac{k t^{n}}{\phi \mu C_{t i}^{n} r_{w}^{2}} .
$$

Where $v$ : seepage velocity, $\mathrm{m} / \mathrm{s} ; k$ : permeability, $\mu \mathrm{m}^{2} ; \mu$ : viscosity, $\mathrm{mPa} \cdot \mathrm{s} ; \frac{\partial P}{\partial r}$ : pressure gradient, $\mathrm{MPa} / \mathrm{m} ; \eta$ : start-up pressure gradient, $\mathrm{MPa} / \mathrm{m} ; V_{E}$ : isothermal adsorption quantity, $\mathrm{m}^{3} / \mathrm{m}^{3} ; V_{L}$ : Langmuir volume; $P_{L}$ : pressure corresponding to $50 \%$ of maximum absorption gas content, MPa; $P_{g}$ : gas pressure, MPa; $V_{m}$ : average absorption gas content, $\mathrm{m}^{3} / \mathrm{m}^{3}$; $D_{m}$ : diffusion coefficient; $F_{s}$ : shape factor; $\pi$ : absorption time constant; $q$ : diffusion amount, $\mathrm{m}^{3} /\left(\mathrm{m}^{3} \cdot \mathrm{d}\right) ; F_{G}$ : geometrical factor; $b$ : slipping coefficient; $h$ : thickness, $\mathrm{m} ; M$ : shale gas molar mass; $p_{m}$ : matrix pressure, $\mathrm{MPa} ; p_{f}$ : fracture pressure , $\mathrm{MPa} ; Q$ : production, $\mathrm{MPa} ; p_{w}$ : bore pressure, $\mathrm{MPa} ; p_{e}$ : boundary pressure, $\mathrm{MPa} ; R$ : general gas constant, getting $0.008314 \mathrm{MPa} \cdot \mathrm{m}^{3} /(\mathrm{kmol} \cdot \mathrm{k}) ; r_{e}$ : supply radius, $\mathrm{m} ; r_{w}$ : bore radius, $\mathrm{m} ; r$ : the distance between the center of wellbore and some point of formation; $S$ : skin factor; $T$ : absolute temperature, $\mathrm{K} ; t$ : production time, $d$; Z: compressibility factor; $\phi$ : porosity; $N$ : total grid number; $n$ : time step; subscript $s c, D$ : respectively representing standard conditions and dimensionless quantity.

\subsection{Verification of the Model and Error Analysis}

In order to evaluate the predicting precision of the model, equation (23) is programmed. Taking a shale gas well as an example, predicting precision of this model is evaluated based on programming calculation. As we can see from Fig.

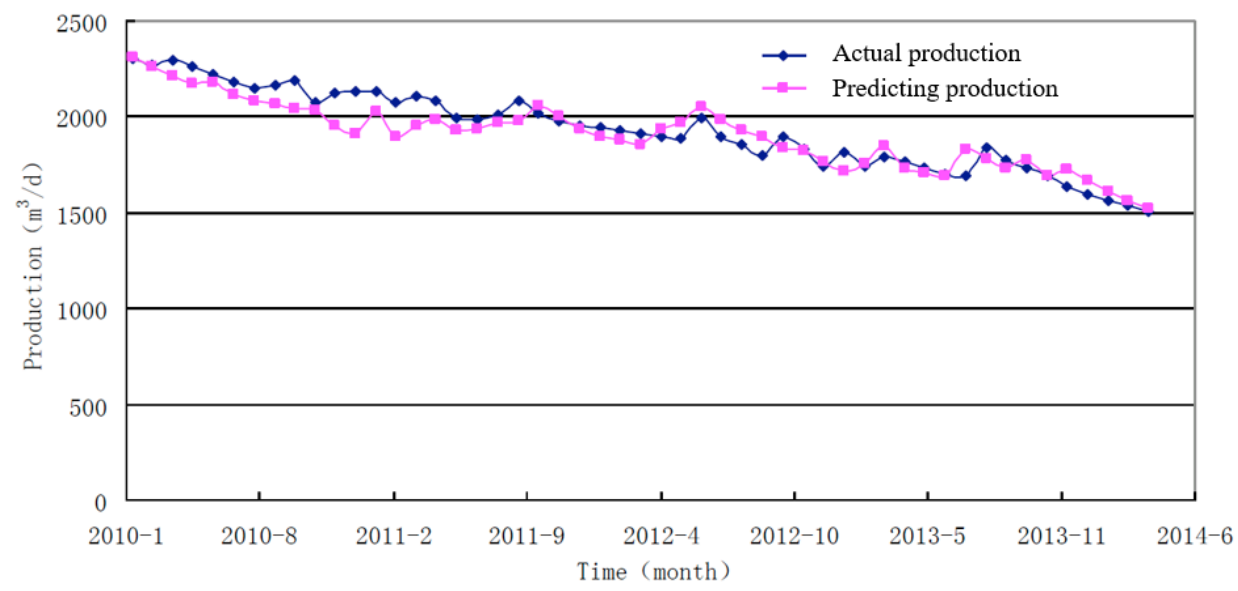

Fig. (1). The result of predicting production. 
(1), predicting curve and the curve of practical data of productivity match well, and the average relative error is $3.82 \%$. There are 3 main reasons for the error: $\ominus$ the basic parameters controlled by a shale gas well, such as thickness, porosity, etc., are denoted with the mean values; $\ominus$ the permeability of artificial fractures and natural fractures cannot be clearly distinguished; $\circledast$ there are differences in the conductivity of the artificial fractures in different grids. In the application of the model, the accuracy and authenticity of all the parameters should be strictly controlled. In this way, the results can meet the practical requirements of engineering.

\section{INFLUENCE FACTOR}

\subsection{Slipping Effect Influence}

Fig. (2) shows the IPR curves corresponding to different slip coefficients. It indicates that at the same pressure difference, as the slip coefficient increases, well production increases. When flow pressure is relatively low, production is greatly influenced by slippage; while the influence is not obvious when the flow pressure is relatively high.

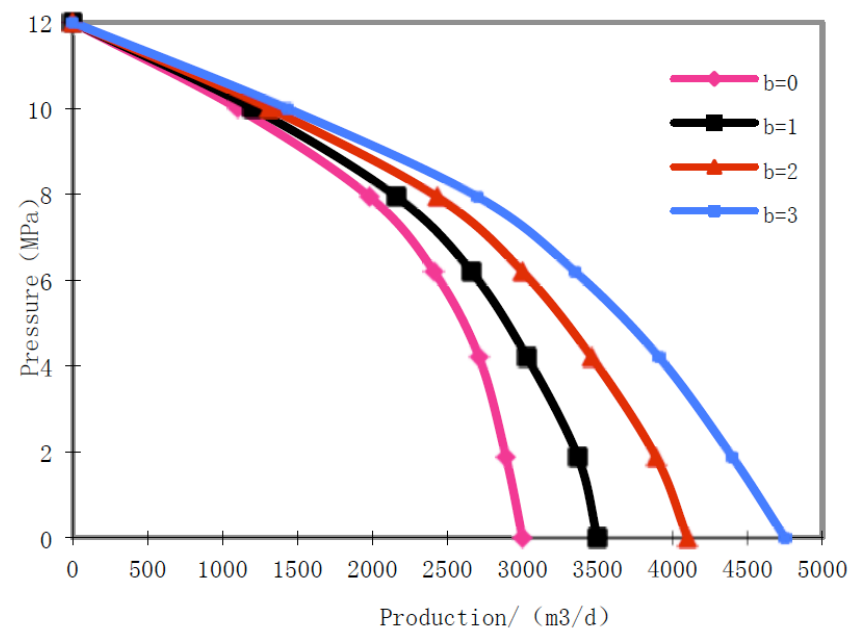

Fig. (2). IPR curve for fracturing wells in different slip coefficients.

\subsection{Start-up Pressure Gradient}

Fig. (3) shows the influence of the start-up pressure gradient on production. It indicates that as the start-up pressure gradient increases, the gas well production decreases. Only when production pressure difference is greater than the startup pressure difference, can gas wells produce gas. This can be verified by the field practice that a start-up pressure difference must be overcome for gas well resuming production after shutting.

\subsection{The Langmuir Pressure Influence}

Fig. (4) shows the influence of the Langmuir pressure on production. It indicates that at the same Langmuir volume, as the Langmuir pressure increases, the pressure spread slows down, and the decrease of production slows down.

\subsection{The Langmuir Volume Influence}

Fig. (5) shows the influence of the Langmuir volume on production. It indicates that at the same Langmuir pressure, as the Langmuir volume increases, the pressure spread slows down. Further contrast indicates that the Langmuir volume influence is linear, whereas the Langmuir pressure influence is nonlinear.

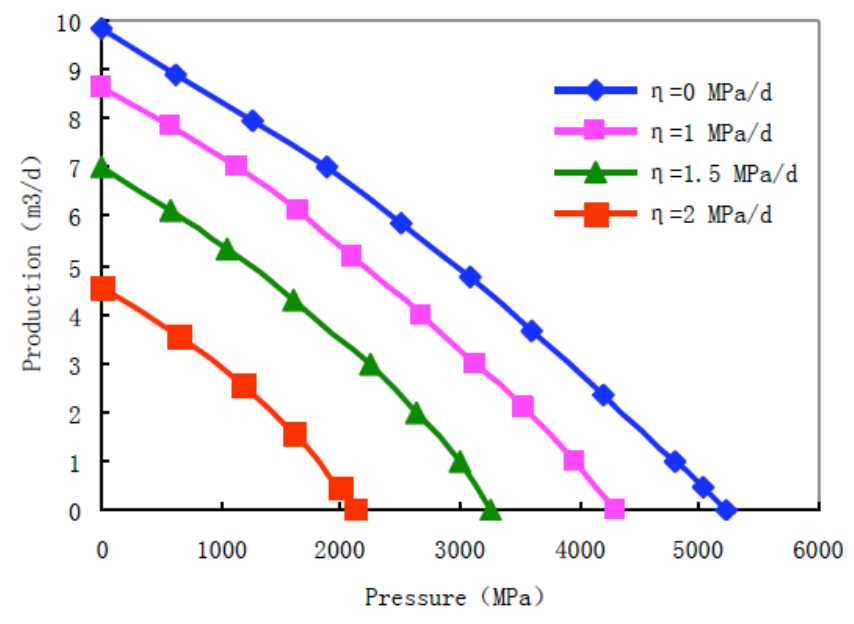

Fig. (3). The influence of start-up pressure gradient.

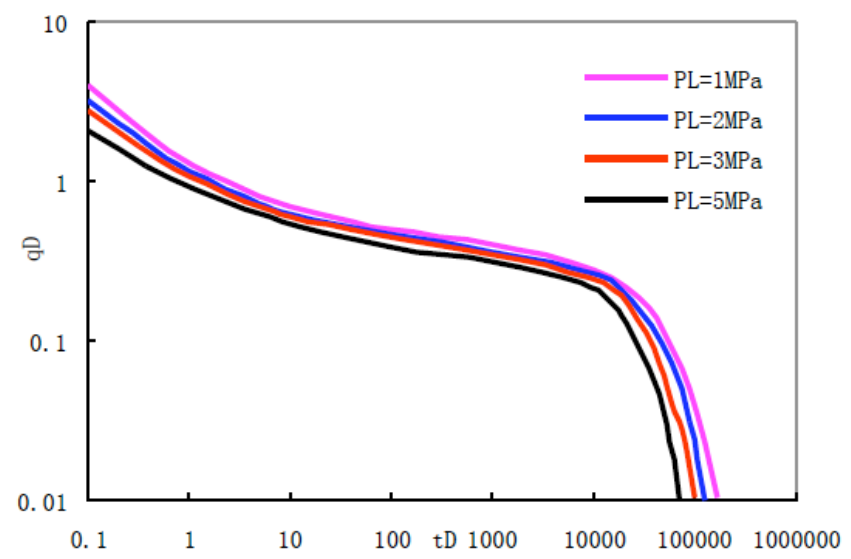

Fig. (4). The influence of Langmuir pressure.

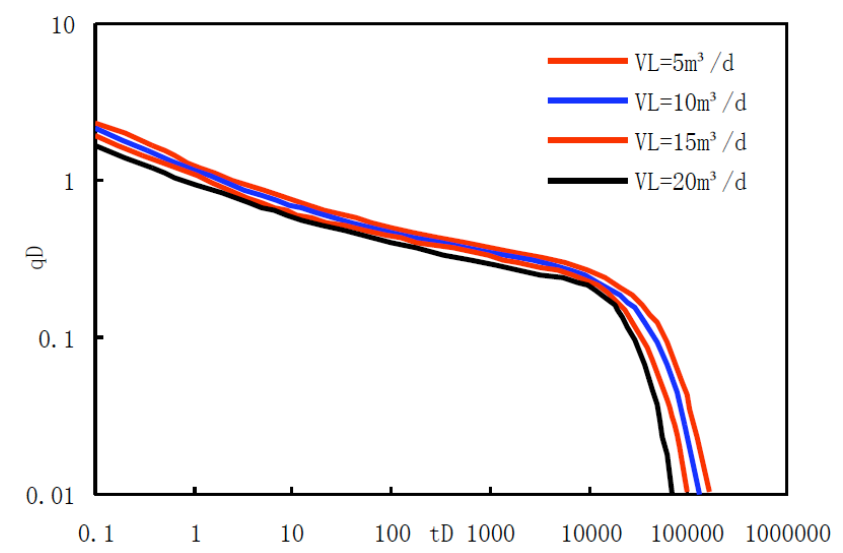

Fig. (5). The influence of Langmuir volume.

\section{CONCLUSION}

[1] Based on start-up pressure gradient, slippage effect and the isothermal adsorption principle, productivity predicting mathematical model of the dual pore medium shale gas reservoir is set up. It can accurately describe desorp- 
tion process, crossflow between matrix and fracture systems and low speed non-Darcy seepage in the fracture system. In addition it can also more closely reflect the practical conditions of the formation.

[2] The results of a shale gas well show that the productivity predicting mathematical model can meet the practical requirements of engineering with a relative error of less than $5 \%$.

[3] The influence of Langmuir volume is linear, whereas the influence of Langmuir pressure is nonlinear.

\section{CONFLICT OF INTEREST}

The authors confirm that this article content has no conflict of interest.

\section{ACKNOWLEDGEMENTS}

This work was financially supported by the Natural Science Foundation of China under Grant (No. 51474071).

\section{REFERENCES}

[1] G. Wang, R. Jiang, and J. Xu, "Analysis and advice for shale gas development”, Complex Hydrocarbon Reservoirs, vol. 5, no. 2, pp. $10-14,2012$.

[2] C. Zhang, and M. Liu, "Cost and benefit analysis of desulfurization system in power plant", Telkomnika (Telecommunication Computing Electronics and Control), vol. 12, no. 1, pp. 33-46, 2014.

[3] R.G. Loucks, R.M. Reed, and D.M. Jarvie, "Morphology, genesis and distribution of nano-scale pores in siliceous mudstones of the Mississippian Barnett shale", Journal of Sedimentary Research, vol. 79, no. 12, pp. 848-861, 2009.
[4] F. Javadpour, D. Fisher, M. Unsworth, "Nanoscale gas flow in shale gas sediments", One Petro., vol. 46, no. 10, pp. 16-21, 2007.

[5] M. Wei, Y. Duan, Q. Fang, Y. Cao, and R. Wang, "Current research situation of porosity \& permeability characteristics and seepage mechanism of shale gas reservoir", Reservoir Evaluation and Development, vol. 1, no. 4, pp. 73-77, 2011.

[6] X. Xu, M. Zhou, X. Wei, C. Kong, X. Huang, and K. Yang, "Analysis on shale gas production affecting factors", Petrochemical Industry Application, vol. 32, no. 9, pp. 10-13, 2013.

[7] G. Ali, M. Dessoky, and A. Hassan, "Comparison between oil immersed and SF6 Gas: power transformers ratings", Telkomnika, vol. 10, no. 1, pp. 43-54, 2012

[8] E. Fathi, and I.Y. Akkutlu, "Matrix heterogeneity effects on gas transport and adsorption in coalbed and shale gas reservoirs", Transport in Porous Media, vol. 80, no. 2, pp. 281-304, 2009.

[9] B. Haghshenas, C.R. Clarkson, and S. Chen, "New models for reserve estimation and non-Darcy gas flow in shale gas reservoirs", Society of Petroleum Engineers - European Unconventional Resources Conference and Exhibition, vol. 2, pp. 1038-1053, 2014.

[10] D.J.K. Ross, and R.M. Bustin, "Characterizing the shale gas resource potential of Devonian Mississippian strata in the western Canada sedimentary basin: application of an integrated formation evaluation", AAPG Bulletin, vol. 92, no. 1, pp. 87-125, 2008.

[11] C. Guo, M. Wei, H. Chen, X. He, and B. Bai, "Improved numerical simulation for shale gas reservoirs", In: Proceedings of the Annual Offshore Technology Conference, vol. 3, pp. 2083-2099, 2014.

[12] F. Ren, X. Wang, and K. Ren, "Characteristics of bottom pressure of shale-gas well considering slippage effect", Journal of Oil and Gas Technology, vol. 35, no. 3, pp. 124-127, 2013.

[13] A. Juan, C. Faruk, and F. Deepak, "Accurate simulation of shalegas reservoirs", The SPE Annual Technical Conference and Exhibition, Florence, vol. 7, pp. 5322-5332, 2010.

[14] J.B. Curtis, "Fractured shale-gas systems,' AAPG Bulletin, vol. 86, no. 11, pp. 1921-1938, 2002

[15] Y. Wei, and Z. Zhao, "Percolation mechanism of shale gas", Liaoning Chemical Industry, vol. 42, no. 2, pp. 152-153, 2013.

Received: April 21, 2015

(C) Daiyin et al.; Licensee Bentham Open.

This is an open access article licensed under the terms of the Creative Commons Attribution Non-Commercial License (http://creativecommons.org/licenses/by-nc/4.0/) which permits unrestricted, non-commercial use, distribution and reproduction in any medium, provided the work is properly cited. 